\title{
Multiphysics of Prionlike Diseases: Progression and Atrophy
}

\author{
Johannes Weickenmeier, ${ }^{1}$ Ellen Kuhl, ${ }^{2}$ and Alain Goriely ${ }^{3}$ \\ ${ }^{1}$ Department of Mechanical Engineering, Stevens Institute of Technology, Hoboken, New Jersey 07030, USA \\ ${ }^{2}$ Living Matter Laboratory, Stanford University, Stanford, California 94305, USA \\ ${ }^{3}$ Mathematical Institute, University of Oxford, Oxford, OX2 6GG, United Kingdom
}

(Received 5 April 2018; revised manuscript received 21 July 2018; published 12 October 2018)

\begin{abstract}
Many neurodegenerative diseases are related to the propagation and accumulation of toxic proteins throughout the brain. The lesions created by aggregates of these toxic proteins further lead to cell death and accelerated tissue atrophy. A striking feature of some of these diseases is their characteristic pattern and evolution, leading to well-codified disease stages visible to neuropathology and associated with various cognitive deficits and pathologies. Here, we simulate the anisotropic propagation and accumulation of toxic proteins in full brain geometry. We show that the same model with different initial seeding zones reproduces the characteristic evolution of different prionlike diseases. We also recover the expected evolution of the total toxic protein load. Finally, we couple our transport model to a mechanical atrophy model to obtain the typical degeneration patterns found in neurodegenerative diseases.
\end{abstract}

DOI: $10.1103 /$ PhysRevLett.121.158101

Introduction.-Age-related neurodegenerative disorders are complex and multifaceted pathologies. In many cases, their evolution is closely associated with the progression of particular protein aggregates, commonly referred to as prions [1]. Prions are corruptive templates or infectious seeds that initiate a chain reaction of protein misfolding and aggregation, creating conformational autocatalysis [2]. Over time, these pathogenic protein assemblies grow, fragment, and spread, preventing proper cell function. Unless they are removed, the accumulation of toxic proteins disrupts the proper function of the nervous system and causes necrosis, brain atrophy, and ultimately death [3]. The concept of prionlike growth, fragmentation, and spreading of toxic proteins has been put forward as the key to Alzheimer's disease, Parkinson's disease, and amyotrophic lateral sclerosis $[4,5]$. Similar mechanisms could also explain secondary injuries following traumatic brain injury [6]. For instance, it is known that the amyloid- $\beta$ protein can form aggregates with a distribution of sizes from small soluble groups to large insoluble fibrils. Their accumulation as plaques is closely associated with Alzheimer's disease. Tau proteins also play an important role in these diseases [7]. Tau is known to stabilize microtubules within the axon; however, when hyperphosphorylated, it forms neurofibrillary tangles that can act as seeds for further misfolding and aggregation. Tau aggregates are found within the axon and

Published by the American Physical Society under the terms of the Creative Commons Attribution 4.0 International license. Further distribution of this work must maintain attribution to the author(s) and the published article's title, journal citation, and DOI. are rapidly transported by interneuronal transport mechanisms [8]. They are also transported into the extracellular space where they diffuse through secretion and damage of the host cell and into cells by endocytosis [9]. Protein aggregates are subject to biological clearance slowing down or arresting the overall spreading process. Neuronal pathways may provide an explanation for the observation that the spreading of neurofibrillary tangles follows a characteristic topographic pattern.

Independent of their molecular origin or specific actions prionlike diseases share the same macroscopic spatiotemporal evolution [10]. The progression is believed to include the following steps: (i) seeding of misfolded proteins, (ii) templated misfolding and aggregation of native proteins, (iii) growth of aggregates, (iv) fragmentation and spatial propagation of aggregates of different sizes, (v) assembly of misfolded proteins into secondary structures (protofibrils and fibrils), and (vi) formation of larger tertiary structures (lesions) at the tissue level. A striking feature of each particular pathology is its systematic evolution pattern. We posit that the main reason for such reproducibility is that the overall progression of the disease is governed by generic transport processes and that the key difference between individual diseases, aside from the particular set of proteins involved, is the original location of the seeds, leading to a spatiotemporal evolution (Fig. 1) characteristic for (a,b) Alzheimer's disease, (c) Parkinson's disease, and (d) amyotrophic lateral sclerosis.

An essential feature of the dynamics is that proteins are preferentially transported transynaptically and can diffuse from the extracellular space to the cells [11]. These effects lead to fast transport along neuronal pathways and slow diffusion away from the axon bundles. Here, we couple an 


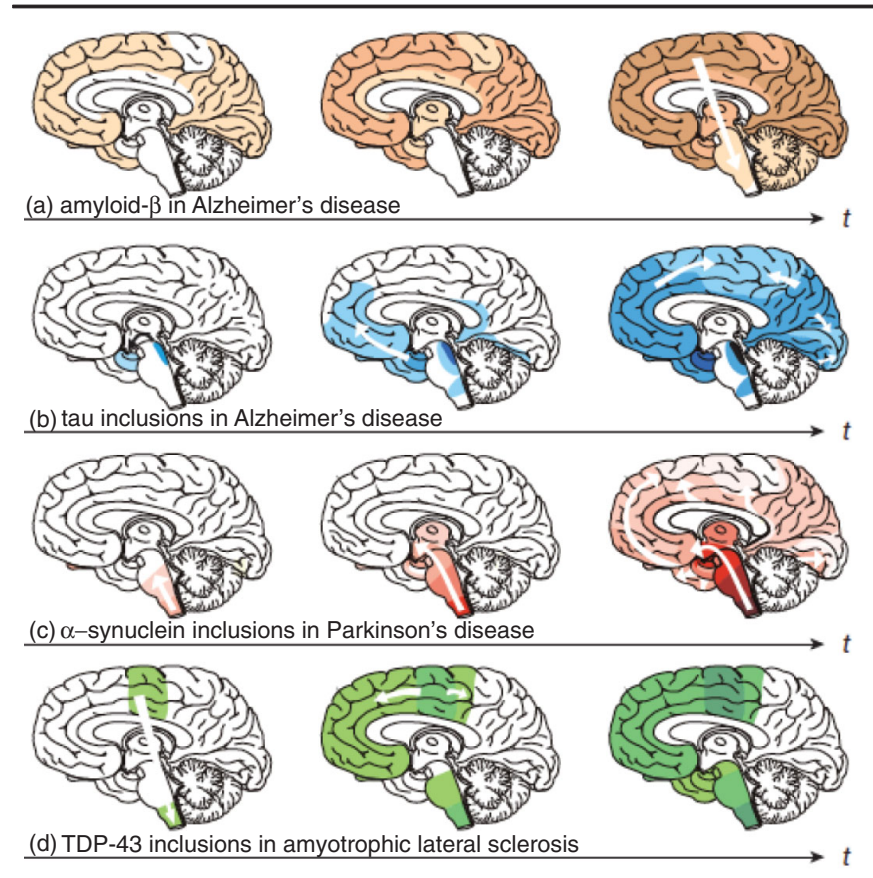

FIG. 1. Typical spatial progression of protein aggregates in various neurodegenerative diseases (from Jucker and Walker [1]).

anisotropic transport process with a mechanical atrophy model to study the relative effects of seed location, anisotropy, and brain geometry on the toxic protein concentration and the resulting atrophy of brain tissue $[12,13]$. We show that a minimal model for transport and atrophy is sufficient to recover the main qualitative spatial features of different neurodegenerative diseases as well as the characteristic overall increase of key biomarkers.

The model.-We couple a transport model to a mechanical model. The brain is taken to be a connected domain $\Omega$ in either $\mathbb{R}^{2}$ (2D simulations) or $\mathbb{R}^{3}$ (3D simulations) with volume $V$, composed of gray $\left(\Omega_{\text {gray }}\right)$ and white $\left(\Omega_{\text {white }}\right)$ matter with distinct material properties. Different models for the aggregation and propagation process have been proposed ranging from graph Laplacian diffusion on simplified structural networks [14] to Smoluchowski-type equations $[15,16]$ tracking the aggregate-size distribution. Here, we use a minimal model for the propagation of a toxic protein that captures the basic characteristics of the problem, for which the concentration $c(\mathbf{x}, t) \in \Omega_{\text {white }}$ follows the FisherKolmogorov-Petrovsky-Piskunov equation

$$
\frac{\partial c}{\partial t}=\nabla \cdot(\mathbf{D} \nabla c)+\alpha c(1-c),
$$

where $\mathbf{D}=d_{\perp} \mathbf{1}+\left(d_{\|}-d_{\perp}\right) \boldsymbol{\gamma} \otimes \boldsymbol{\gamma}$ is a transversely anisotropic diffusion tensor with a preferential direction along the axon bundle characterized by the unit vector $\gamma=\gamma(\mathbf{x}, t), \alpha>0$ is the growth rate, $d_{\perp}$ is the tissue diffusion, and $d_{\|} \gg d_{\perp}$ is the diffusion along the axons. This model describes the overall increase of toxic protein concentration, assuming that the pool of proteins in the native form is sufficiently large. The nonlinearity provides a saturation term expressing the maximal concentration of toxic proteins (taken to be one without loss of generality). In one dimension (along the axonal bundles), the system supports traveling fronts with a velocity $v=$ $2 \sqrt{\alpha d_{\|}}$leading to a complete invasion in a characteristic time $T=L / v$, where $L$ is a length scale for the brain, which is on the order of $16 \mathrm{~cm}$ in humans. In the gray matter, for $\mathbf{x} \in \Omega_{\text {gray }}$, we assume pure isotropic diffusion [Eq. (1) with $d_{\|}=d_{\perp}$ and $\alpha=0$ ].

We track two quantities related to the concentration: the time $\tau\left(\mathbf{x}, C_{\text {crit }}\right)$ at which the concentration first reaches a critical level close to the saturation level $C_{\text {crit }}=(1-\varepsilon)$, defined implicitly by $c(\mathbf{x}, \tau)=C_{\text {crit }}$, and the "biomarker abnormality" defined as

$$
C(t)=\frac{1}{V} \int_{\Omega} c(\mathbf{x}, t) d \mathbf{x}
$$

We couple transport to atrophy by assuming that brain tissues are morphoelastic materials $[17,18]$ and assigning an isotropic shrinking factor $0<\vartheta \leq 1$ at each point, depending on the concentration of toxic proteins. Given an initial reference configuration, atrophy is characterized by a deformation $\mathbf{x}(\mathbf{X}, t)=\boldsymbol{\varphi}(\mathbf{X}, t)$ from the reference brain $\Omega$ to the aged brain after shrinking $\Omega_{t}$. Here, $\mathbf{x}(\mathbf{X}, t)$ is the position at time $t$ of the material point originally located at $\mathbf{X}$ at time $t=0$. This deformation defines the deformation gradient $\mathbf{F}=\nabla_{\mathbf{X}} \boldsymbol{\varphi}$ and we assume that it has the form $\mathbf{F}=\vartheta^{1 / 3} \mathbf{A}$. The elastic deformation tensor $\mathbf{A}$ is computed by solving the Cauchy equation $\operatorname{div}(\mathbf{T})=\mathbf{0}$ for the stress tensor $\mathbf{T}=J^{-1} \mathbf{A} \cdot \partial_{\mathbf{A}} W$ associated with the strain-energy density $W=W(\mathbf{A}, \mathbf{x})$ and $J=\operatorname{det}(\mathbf{A})$. The relative rate of shrinking is proportional to the total exposure of the tissue to the toxic proteins, so that $\partial_{t} \vartheta=-\delta c \vartheta$, where $\delta>0$ is an overall parameter that describes the rate of material removal.

Computational investigation.-We create finite element models from $\mathrm{T} 2$-weighted magnetic resonance images of a 32-yr-old male using Simpleware (Synopsis Inc., Mountain View, CA): a two-dimensional sagittal model, a two-dimensional coronal model, and a fully three-dimensional, anatomically accurate whole brain model. The sagittal model has 13442 linear triangular elements and 7216 nodes; the coronal model has 38223 linear triangular elements and 19813 nodes; and the three-dimensional whole brain model has 401940 linear tetrahedral elements and 80233 nodes. We tested different levels of mesh refinement and found that these discretizations provide the best balance between computational cost, efficiency, and accuracy. To model anisotropic toxic protein propagation, we register the axonal fiber orientation $\gamma$ from diffusion tensor magnetic resonance images and create an element-based fiber-orientation map. 
For the initial seeding, we assume a concentration $c(\mathbf{x})=1$ for all $\mathbf{x} \in \Omega_{\text {seed }} \subset \Omega$ and $c(\mathbf{x})=0$ otherwise. Then, using Eq. (1) we propagate the toxic protein across the brain with a ratio of $d_{\|}: d_{\perp}=100$ to ensure a faster propagation along the axonal fiber direction. In the white matter, we use $d_{\|}=100 \mathrm{~mm}^{2} / \mathrm{yr}$ and $\alpha=0.5 / \mathrm{yr}$. In the gray matter, we use $d_{\|}=d_{\perp}=10 \mathrm{~mm}^{2} / \mathrm{yr}$.

Motivated by Fig. 1, we postprocess the concentration field $c$ to extract the activation time $\tau\left(\mathbf{x}, C_{\text {crit }}\right)$. From those activation times, we create the activation maps corresponding to Fig. 1. At any given time $t$, we display activation time a single color and a linear shading intensity between 0 and 1 that is equal to $(t-\tau) / t$ for $\tau<t$ and zero for all $\tau \geq t$ (the shading is maximal in the initial seeding zone and there is no shading in nonactivated regions with $\tau \geq t$ ). Using Eq. (2), we compute the biomarker abnormality $C(t)$ to create a temporal map of the toxic protein concentration averaged over the entire brain [19].

Finally, using the tissue atrophy model with different atrophy rates $\delta$ in the gray and white matter tissue, we use our two-dimensional coronal model to create atrophy maps. Since atrophy takes place primarily in locations with elevated concentrations of toxic proteins, the change of volume does not significantly affect the time evolution of the concentration since it is already close to maximal in these locations. We take advantage of this property to compute tissue shrinking as a postprocessing step based on the values of the concentration at different time points. Since we assume that, after each step of shrinking, there is total relaxation of the stresses, the mechanical stresses generated are small during shrinking and we can use a standard compressible neo-Hookean model valid in small deformations with parameters fitted to experimental data: $W=c_{1}\left[\operatorname{tr}\left(\mathbf{A}^{\mathrm{T}} \cdot \mathbf{A}\right)-3\right]+c_{2}(J-1)^{2}$ with $c_{1}=666.56 \mathrm{~Pa}$ and $c_{2}=1777.5 \mathrm{~Pa}$ [20]. For the relative shrinking rate, it is known that the gray matter tissue shrinks and loses more volume than the white matter tissues [21] and we use a ratio $\vartheta_{\text {gray }}: \vartheta_{\text {white }}=4: 1$.

$2 D$ simulations. - We begin with $2 \mathrm{D}$ simulations by restricting our domain to a sagittal slice (Fig. 2). The region includes the ventricle but, through no-flux boundary conditions, it is assumed that no progression takes place into this region. Based on the description of the evolution of tau inclusions in Alzheimer's disease, we place an initial seeding of toxic protein close to the entorhinal cortex [Fig. 2(a)]. We calculate the spatiotemporal evolution of the toxic protein concentration and extract the activation map in Fig. 2. We observe the quick progression into the brain stem and the hippocampus [Fig. 2(a)], to paralimbic and adjacent medial-basal temporal cortex [Fig. (2b)], to cortical association areas [Fig. 2(c)], and eventually reaching primary sensory-motor and visual areas. The relatively rapid progression along the hippocampus is associated with a strong anisotropy along the axonal pathways tangent to
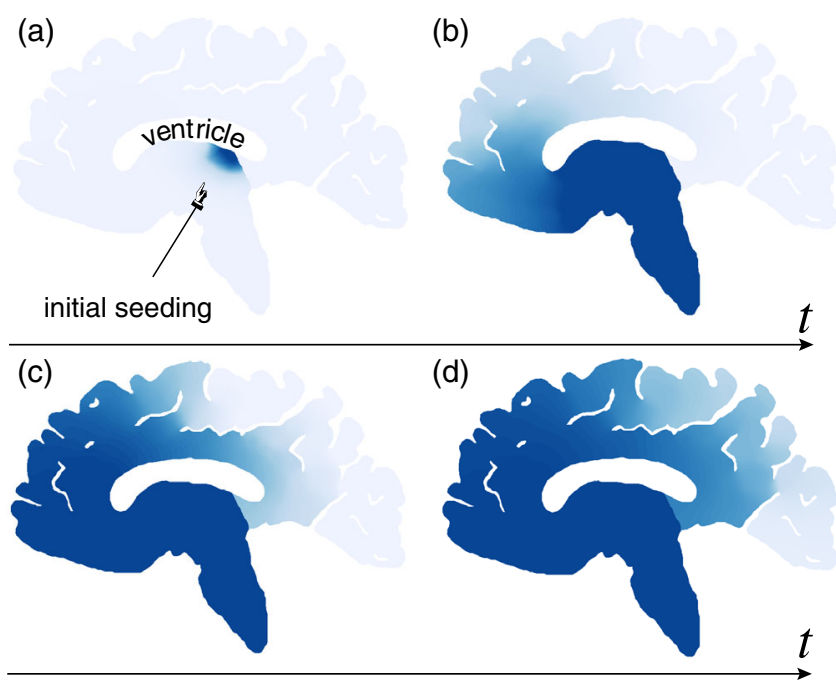

FIG. 2. Activation maps of tau inclusions in brains of patients with Alzheimer's disease simulated across a 2D sagittal slice at time $t=0,5,10,15$ (yr), Figs. 2(a)-(d) respectively.

the ventricles. This progression is consistent with the wellestablished Braak stages of Alzheimer's disease [22]: stages I and II "transentorhinal" [Fig. 2(a)]; stages III and IV "limbic" [Fig. 2(b)]; stages V and VI "isocortical" [Figs. 2(c) and 2(d)].

$3 D$ simulations. - The $2 \mathrm{D}$ analysis uses a high-resolution model, but ignores the truly 3D character of the brain. To study the effect of the initial seeding regions of amyloid- $\beta$, tau, $\alpha$-synuclein, and TDP-43, we prescribe a nonzero toxic protein concentration as an initial condition, $c(\mathbf{x}, t=0)>0$, in the four seeding regions illustrated in Fig. 1, while keeping the geometry and all other parameters the same for all four simulations. We then advance the system in time, calculate the spatiotemporal evolution of the toxic protein concentration, extract the four activation maps in Fig. 3, and compare them against the literature [1]. We observe generic progression trends in all four cases. (i) As soon as a sufficient level of toxic proteins reaches the area close to the ventricles, there is a fast progression in the limbic system (around the ventricles) leading to a rapid invasion of the temporal and occipital lobes. (ii) Once a sufficient level of toxic protein is reached within the cortex, further invasion through the cortex takes place. (iii) If the parietal lobe is not directly involved initially, it only becomes invaded in the last stages of the disease.

An indirect way to follow the evolution of neurodegenerative diseases is to look at the averaged concentration of toxic proteins or associated biomarkers. The paradigm for this evolution is based on the so-called Jack's curves. These curves are the expected, mostly hypothetical, evolution of a typical biomarker concentration as a function of time [23]. They take the shape of sigmoidlike functions: a slow evolution at first during incubation, followed by a sharp increase during the outbreak, and leading to saturation when the disease is fully established $[16,19]$. The variation 


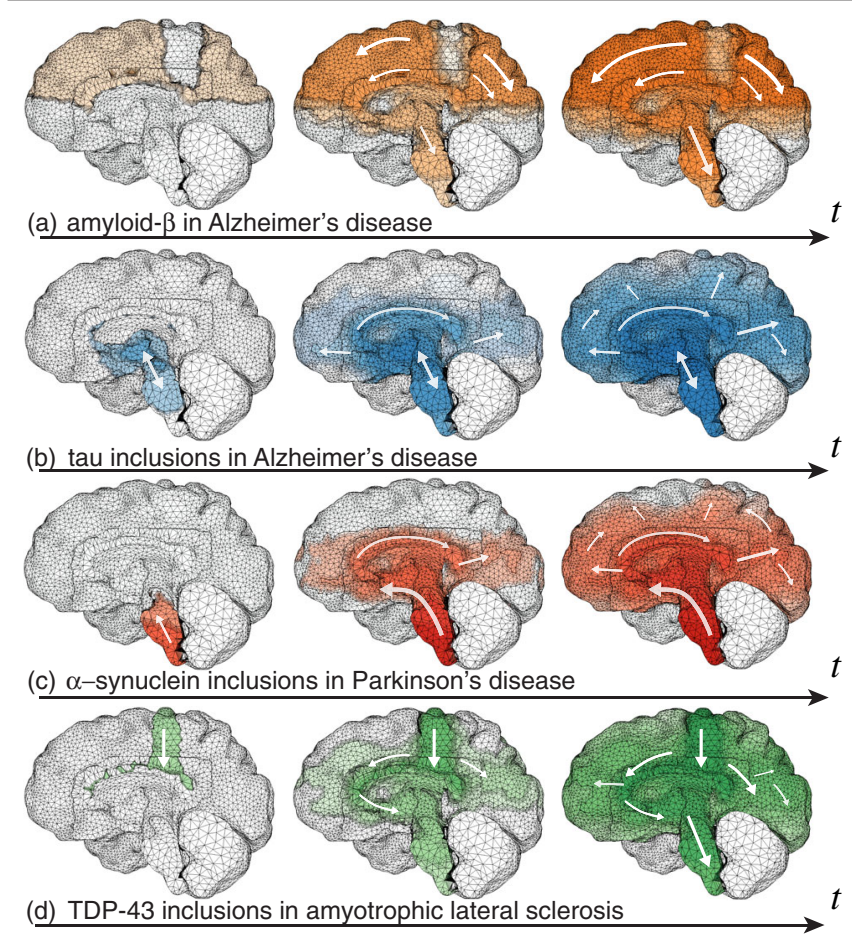

FIG. 3. Activation maps for the spatial progression of toxic protein for various initial seeding regions, associated with $(a, b)$ Alzheimer's disease, (c) Parkinson's disease, and (d) amyotrophic lateral sclerosis, simulated across a 3D brain at time $t=0,10,20$ (yr), left to right column respectively.

of the typical parameters entering the sigmoids are used to represent physiological quantities such as time of outbreak and rate of spreading. These curves serve as a general guideline to understand the variations between individuals, pathologies, and the effect of various stimuli or therapeutics [24]. We can extract these curves from the simulation using (2), as shown in Fig. 4. Our simulations recover the predicted behavior for this type of disease integrated over the entire brain with typical timescales similar to the ones obtained from front solutions of Eq. (1) in one dimension. Indeed, using the typical wave speed of front propagation in one dimension, we obtain a lower and upper bound for the total invasion time of 11.3 and $35.8 \mathrm{yr}$ (using either the fast axonal diffusion constant $d_{\|} \approx 100 \mathrm{~mm}^{2} / \mathrm{yr}$ or the slow gray matter diffusion constant $d \approx 10 \mathrm{~mm}^{2} / \mathrm{yr}$ ). Figure 4 also highlights a parametric study of the main parameters in Eq. (1) with the 2D baseline parameters (blue), a twofold increase of the growth parameter $\alpha$ (green), a fourfold increase of isotropic diffusion through the parameters $d_{\perp}$ and $d_{\|}$(orange), and an eightfold increase of only the anisotropic parameter $d_{\|}$(red). We observe an interesting trend for the "outbreak time," defined as the time at which the biomarker abnormality reaches a certain level $1 / 2<C_{\text {outbreak }}<1$. The outbreak time decreases when the growth rate $\alpha$ increases. But, counterintuitively, the outbreak time increases with the diffusion coefficients. This is

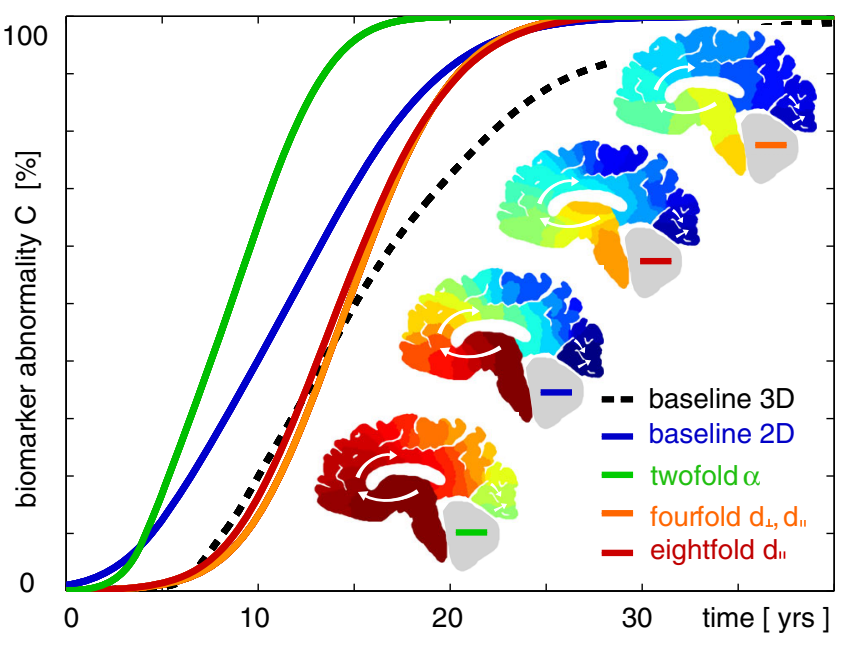

FIG. 4. Biomarker abnormality $C$ as the concentration $c$ integrated over the 3D brain (dashed line) and 2D sagittal slice (solid lines). Parametric study with 2D baseline parametrization (blue), twofold growth $\alpha$ (green), fourfold diffusion $d_{\perp}$ and $d_{\|}$ (orange), and eightfold diffusion $d_{\|}$along the axons (red) compared to 3D brain (dashed black).

due to the fact that diffusion tends to spread out the toxic protein concentrations over the entire brain, hence reducing the effect of growth. However, once initiated, the biomarker $C$ increases more rapidly with increasing $\alpha$ and $d$ (as indicated by the slope of the curves), which is in line with a faster spreading of toxic proteins as predicted by $v=2 \sqrt{\alpha d_{\|}}$. While both parameters $\alpha$ and $d$ have similar effects in an integral sense, the concentration maps of the four different parametrizations in Fig. 4 clearly show distinct activation patterns. Specifically, increasing the anisotropic diffusion $d_{\|}$(second concentration map) triggers a faster spreading of toxic proteins along the fiber direction around the ventricle. The fully $3 \mathrm{D}$ brain model (dashed black) follows the same global trend of a progressive invasion [24].

Atrophy.-We now turn to the effect of elevated toxic proteins on the tissue. It is known that the formation of large aggregates or high concentration of toxic proteins prevents the proper function of neuronal cells, leading to ischemia and eventually tissue removal. For instance, in the top row of Fig. 5, there is a marked and rapid atrophy associated with the disease. We use our propagation model for the coronal slice to compute the toxic protein concentration at four consecutive points in time (middle row). At each time point, we simulate the toxic protein-induced atrophy across the coronal slice. Our simulated atrophy patterns (bottom row) agree well with the atrophy pattern observed in Alzheimer's disease (top row): they display pronounced hippocampal atrophy, ventricular enlargement, and a widening of the cortical sulci and suggest that pronounced atrophy is a result of high toxic protein concentrations. 


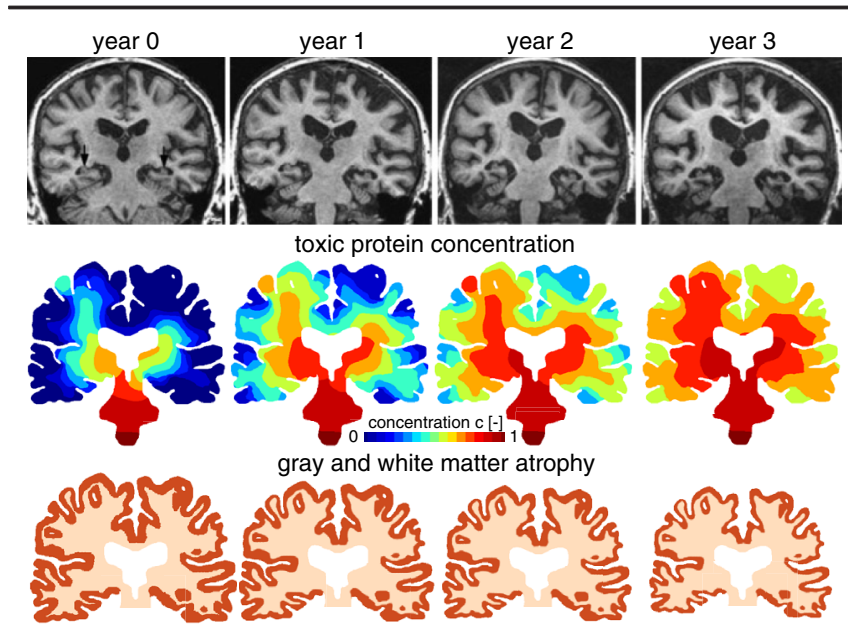

FIG. 5. Atrophy. (Top) Magnetic resonance images of Alzheimer's patient; annual examination reveals increasing hippocampal atrophy, ventricular enlargement, and widening of the cortical sulci [25]. (Middle) Annual evolution of toxic protein concentration based on an initial seeding in the brain stem. (Bottom) Simulated annual atrophy patterns based on activation time and toxic protein concentration with an atrophy ratio of $\vartheta_{\text {gray }}: \vartheta_{\text {white }}=4: 1$.

Conclusions.-Neurodegenerative diseases are known for their extreme complexity, and medical science has wrestled with this major challenge by amassing a considerable amount of information, despite few therapeutic successes. One of the remarkable features that appear in these diseases is their highly reproducible topographic propagation pattern. Each disease has a well-defined spatiotemporal evolution that has been documented over the years and cataloged into stages, each associated with typical symptoms. This evolution appears much more controlled than other diseases such as cancer and has led to three main hypotheses in recent years. First, it has been proposed that diseases share the same characteristics as prion diseases and are based on the seeding, propagation, and accumulation of toxic proteins involved in regular subcellular functions such as $\alpha$ synucleins or tau proteins. Second, it has been proposed that the overall evolution of a typical biomarker follows regular sigmoidlike curves. Third, a given disease is associated with typical atrophy patterns.

There have been multiple mathematical models proposed for neurodegenerative diseases, but most of them focus on biochemical pathways, cellular interactions, and the formation of amyloids [26-28]. Our approach here is different as we study the problem at the largest possible scale of the brain. Our minimal model takes into account the main microscopic features of these diseases, but incorporates the full brain geometry and axonal directions from diffusion tensor imaging. Despite the complexity of these diseases, our model recovers the three aforementioned key features of neurodegenerative disease progression and suggests that brain geometry, transport anisotropy, and mechanics play a central role in neurodegeneration and atrophy pattern formation.

This work was supported by the NSF Grant No. CMMI 1727268 to E. K. The support for A. G. by the Engineering and Physical Sciences Research Council of Great Britain under research Grant No. EP/R020205/1 is gratefully acknowledged.

[1] M. Jucker and L. C. Walker, Nature (London) 501, 45 (2013).

[2] S. B. Prusiner, Proc. Natl. Acad. Sci. U.S.A. 95, 13363 (1998).

[3] J. Brettschneider, K. Del Tredici, V. M.-Y. Lee, and J. Q. Trojanowski, Nat. Rev. Neurosci. 16, 109 (2015).

[4] M. Cruz-Haces, J. Tang, G. Acosta, J. Fernandez, and R. Shi, Transl. Neurodegener. 6, 20 (2017).

[5] J. C. Watts, C. Condello, J. Stöhr, A. Oehler, J. Lee, S. J. DeArmond, L. Lannfelt, M. Ingelsson, K. Giles, and S. B. Prusiner, Proc. Natl. Acad. Sci. U.S.A. 111, 10323 (2014).

[6] A. C. McKee, T. D. Stein, C. J. Nowinski, R. A. Stern, D. H. Daneshvar, V. E. Alvarez, H.-S. Lee, G. Hall, S. M. Wojtowicz, C. M. Baugh et al., Brain 136, 43 (2013).

[7] M. Goedert, Science 349, 1255555 (2015).

[8] P. C. Bressloff and J. M. Newby, Rev. Mod. Phys. 85, 135 (2013).

[9] C. Soto, Neuron 73, 621 (2012).

[10] L. C. Walker and M. Jucker, Annu. Rev. Neurosci. 38, 87 (2015).

[11] B. Frost, R. L. Jacks, and M. I. Diamond, J. Biol. Chem. 284, 12845 (2009).

[12] A. Goriely, M. G. D. Geers, G. A. Holzapfel, J. Jayamohan, A. Jérusalem, S. Sivaloganathan, W. Squier, J. A. W. van Dommelen, S. L. Waters, and E. Kuhl, Biomech. Model. Mechanobiol. 14, 931 (2015).

[13] A. Goriely, S. Budday, and E. Kuhl, Adv. Appl. Mech. 48, 79 (2015).

[14] F. Abdelnour, H. U. Voss, and A. Raj, NeuroImage 90, 335 (2014).

[15] P. C. Bressloff, Lecture Notes on Mathematical Modelling in the Life Sciences (Springer, New York, NY, 2014), DOI: $10.1007 / 978-1-4614-8866-8$.

[16] M. Bertsch, B. Franchi, N. Marcello, M. C. Tesi, and A. Tosin, Math. Med. Biol. 34, 193 (2016).

[17] E. Kuhl, J. Mech. Behav. Biomed. Mater. 29, 529 (2014).

[18] A. Goriely, The Mathematics and Mechanics of Biological Growth (Springer Verlag, New York, 2017).

[19] C. R. Jack and D. M. Holtzman, Neuron 80, 1347 (2013).

[20] L. A. Mihai, L. Chin, P. A. Janmey, and A. Goriely, J. R. Soc. Interface 12, 20150486 (2015).

[21] P. M. Thompson, K. M. Hayashi, G. De Zubicaray, A. L. Janke, S. E. Rose, J. Semple, D. Herman, M. S. Hong, S. S. Dittmer, D. M. Doddrell et al., J. Neurosci. 23, 994 (2003).

[22] H. Braak and E. Braak, Acta Neuropathol. 82, 239 (1991).

[23] C. R. Jack Jr., D. S. Knopman, W. J. Jagust, R. C. Petersen, M. W. Weiner, P. S. Aisen, L. M. Shaw, P. Vemuri, H. J. Wiste, S. D. Weigand et al., Lancet Neurol. 12, 207 (2013). 
[24] C. R. Jack Jr., D. S. Knopman, W. J. Jagust, R. C. Petersen, M. W. Weiner, P. S. Aisen, L. M. Shaw, P. Vemuri, H. J. Wiste, S. D. Weigand et al., Lancet Neurol. 12, 207 (2013).

[25] S. Lehéricy, M. Marjanska, L. Mesrob, M. Sarazin, and S. Kinkingnehun, Eur. J. Radiol. 17, 347 (2007).

[26] M. Zamparo, A. Trovato, and A. Maritan, Phys. Rev. Lett. 105, 108102 (2010).
[27] T. C. T. Michaels, S. I. A. Cohen, M. Vendruscolo, C. M. Dobson, and T. P. J. Knowles, Phys. Rev. Lett. 116, 038101 (2016).

[28] A. Lloret-Villas, T. Varusai, N. Juty, C. Laibe, N. Le Novère, H. Hermjakob, and V. Chelliah, CPT: Pharmacometrics Syst. Pharmacol. 6, 73 (2017). 